Available on line at www.rac.es/racsam Applied Mathematics
REVISTA DE LA REAL ACADEMIA DE CIENCIAS EXACTAS, FISICAS Y NATURALES.

$\mathbb{S E R I E} \mathbb{A}: \mathbb{M} \mathbb{T} \mathbb{E} M \mathbb{A} \mathbb{T} \mathbb{C} \mathbb{S}$ Madrid (España / Spain)

\title{
About the solution of the even parity formulation of the transient radiative heat transfer equations
}

\author{
Óscar López-Pouso and Rafael Muñoz-Sola
}

\begin{abstract}
This paper studies the existence and uniqueness questions, in classical spaces, for a certain system of differential equations. From the physical point of view, the interest of this system lies in that it becomes, for particular choices of its coefficients, the even parity formulation of the $S_{2}$ approximation of the transient radiative heat transfer equations in the one-dimensional slab. A priori lower and upper bounds of the solution are obtained as well. \section{transferencia de calor por radiación}

Sobre la solución de la formulación par de las ecuaciones evolutivas de

Resumen. Este artículo aborda las cuestiones de existencia y unicidad, en espacios clásicos, para cierto sistema de ecuaciones diferenciales. Desde el punto de vista físico, el interés de este sistema radica en que se convierte, para elecciones particulares de sus coeficientes, en la formulación par de la aproximación $S_{2}$ de las ecuaciones evolutivas de la tranferencia de calor por radiación en una geometría de laja (slab) unidimensional. Asimismo, se obtienen cotas a priori, tanto inferiores como superiores, de la solución.
\end{abstract}

\section{Introduction}

Let us consider the following nonlinear system of equations on the nonvoid bounded domain $\left(t_{0}, t_{f}\right) \times$ $(A, B) \subset \mathbb{R}^{2}$ :

$$
\begin{cases}\frac{\partial T}{\partial t}=\tilde{f}(t, x)-c_{1} T^{4}+c_{2} F & \text { (temperature equation), } \\ -\frac{\partial^{2} F}{\partial x^{2}}+c_{3} F=c_{4} T^{4} & \text { (radiative heat flux equation), }\end{cases}
$$

Submitted by Jesús Ildefonso Díaz

Received: May 26, 2009. Accepted: October 8, 2009

Keywords: Radiative heat transfer, even parity formulation, $S_{2}$ approximation, existence, uniqueness, a priori bounds Mathematics Subject Classifications: 35A01, 35A02, 35G61, 47J35, 80A20, 85A25

(C) 2010 Real Academia de Ciencias, España 
which is closed with the initial condition

$$
T\left(t_{0}, x\right)=T_{0}(x)
$$

for $x \in(A, B)$, and the boundary conditions

$$
\left\{\begin{array}{l}
F(t, A)-c_{5} \frac{\partial F}{\partial x}(t, A)=l_{A}(t), \\
F(t, B)+c_{5} \frac{\partial F}{\partial x}(t, B)=l_{B}(t)
\end{array}\right.
$$

for $t \in\left(t_{0}, t_{f}\right)$.

Each $c_{i}$, for $i \in\{1,2,3,4,5\}$, is a given positive real number (positive means strictly positive), and $\tilde{f}(t, x), T_{0}(x), l_{A}(t)$, and $l_{B}(t)$ are given real functions. The unknowns are the temperature $T(t, x)$ and the net radiative heat flux $F(t, x)$. Time $t$ belongs to the interval $\left(t_{0}, t_{f}\right)$ (or $\left[t_{0}, t_{f}\right]$ ) and space $x$ belongs to the interval $(A, B)$ (or $[A, B])$.

For particular values of the constants $c_{i}$, the system (1)-(2) is known as the even parity formulation of the $S_{2}$ approximation of the transient radiative transfer equations in the one-dimensional slab (see Equations (2.9) and (2.14) in reference [3], where one can find as well the description of two algorithms for solving that system, together with numerical results).

Equation (1) is a heat equation, with a heat source $\tilde{f}$, where conduction and convection have been neglected, but that brings the effect of the radiation into play, which obliges to include Equation (2). ${ }^{1}$ For situations where the $S_{2}$ approximation is not accurate enough, more complete mathematical models can be consulted in references $[5,7,8]$.

The system models a situation where radiative heat transfer occurs within a participating medium. In this context, the adjective participating indicates that the medium absorbs, emits, and scatters thermal radiation. We note that radiation heat transfer can also take place through non participating media such as the vacuum.

We give in this paper sufficient conditions for existence and uniqueness in classical spaces. Whether the solution is global or local in time depends on the sign of the critical parameter $\gamma=c_{2} c_{4} \mu_{1}-c_{1}$, where $\mu_{1}>0$ is the first eigenvalue of certain self-adjoint operator $\mathcal{L}$ that we shall define later on. Since $\mu_{1}$ depends on $c_{3}$ and $c_{5}$, it is through $\mu_{1}$ that the influence of the parameters $c_{3}$ and $c_{5}$ plays its role. The most relevant conclusion is that when $\gamma \leq 0$, which includes the physically relevant case, a unique global solution exists.

An a priori lower bound of the solution, valid for any value of $\gamma$, is given, and an a priori upper bound valid when $\gamma \leq 0$ is obtained as well.

We notice that in [6] it is studied the multidimensional physical case considering both conduction and radiation. Since we study the situation of radiative equilibrium, we do not include heat conduction and, consequently, the results of this paper cannot be derived from those in [6].

Henceforth, the spaces $\mathrm{C}^{0}\left(\left[t_{0}, t_{f}\right] \times[A, B]\right)$ and $\mathrm{C}^{0}\left(\left[t_{0}, t_{f}\right] ; \mathrm{C}^{0}([A, B])\right)$ will be identified without further explanation, and thus $\psi(t)$ will be a function of $\mathrm{C}^{0}([A, B])$ in case $\psi \in \mathrm{C}^{0}\left(\left[t_{0}, t_{f}\right] \times[A, B]\right)$.

\footnotetext{
${ }^{1}$ Excerpted from [5, Ch. 8]: "Much attention [...] will be given to the situation in which radiation is the dominant mode of heat transfer, meaning that when conduction and convection are negligible. This situation is referred to as radiative equilibrium [...] Radiative equilibrium is often a good assumption in applications with extremely high temperatures, such as plasmas, nuclear explosions, and such."
} 


\section{Tools}

\subsection{The second order ODE. Reformulation}

It is known ([2]) that, for $\sigma$ given in $\mathrm{L}^{1}(A, B)$ and $l_{A}, l_{B}$ given in $\mathbb{R}$, the problem

$$
\left\{\begin{array}{l}
-u^{\prime \prime}+c_{3} u=\sigma \\
u(A)-c_{5} u^{\prime}(A)=l_{A}, \\
u(B)+c_{5} u^{\prime}(B)=l_{B},
\end{array}\right.
$$

has a unique solution $u \in \mathrm{W}^{2,1}(A, B)$, satisfying the ODE at almost every point, which can be represented by the formula

$$
u(x)=\mathcal{K}(\sigma)(x)=\int_{A}^{B} G(x, s) \sigma(s) \mathrm{d} s+h(x),
$$

where $G \in \mathrm{C}^{0}([A, B] \times[A, B])$ is the associated Green function and $h$ is a $\mathrm{C}^{\infty}$ function carrying the information of the boundary conditions in such a way that $h \equiv 0$ if $l_{A}=l_{B}=0$. Note that $c_{3}$ and $c_{5}$ are the constants appearing in Equations (2), (4) and (5), and hence they are positive.

Among the properties of $G$, we single out that $G$ is nonnegative and symmetric $(G(x, s)=G(s, x)$ for all $(x, s) \in[A, B] \times[A, B])$.

Functions $G$ and $h$ are explicitly described in Appendix A.

Moreover, $\mathcal{K}(\sigma) \geq 0$ whenever $\sigma \geq 0, l_{A} \geq 0$, and $l_{B} \geq 0$ hold simultaneously ([2]). Since $h$ does not depend on $\sigma$, we also have, simply by taking $\sigma \equiv 0$, that $h \geq 0$ whenever $l_{A} \geq 0$ and $l_{B} \geq 0$ hold simultaneously.

When $\sigma \in \mathrm{C}^{0}([A, B])$, it turns out that $\mathcal{K}(\sigma) \in \mathrm{C}^{2}([A, B])$ and that the ODE is satisfied at every point. It will also become of particular interest the bound

$$
\left\|\mathcal{K}\left(\sigma_{1}\right)-\mathcal{K}\left(\sigma_{2}\right)\right\|_{\mathrm{C}^{0}([A, B])} \leq(B-A)\|G\|_{\mathrm{C}^{0}([A, B] \times[A, B])}\left\|\sigma_{1}-\sigma_{2}\right\|_{\mathrm{C}^{0}([A, B])},
$$

which is easily derived from Equation (7).

When $l_{A}$ and $l_{B}$ are functions of time $t$, one can apply the previous results at every time $t$ and obtain

$$
u(t, x)=\mathcal{K}(t, \sigma)(x)=\int_{A}^{B} G(x, s) \sigma(s) \mathrm{d} s+h(t, x),
$$

being the time regularity of $h$ (and hence of $u$ ) the minimum of the regularities of functions $l_{A}$ and $l_{B}$. In particular, time continuity is ensured if $l_{A}$ and $l_{B}$ are continuous. Note that the difference $\mathcal{K}\left(t, \sigma_{1}\right)-$ $\mathcal{K}\left(t, \sigma_{2}\right)$ does not depend on $t$ anymore, and the same bound $(8)$ holds for $\left\|\mathcal{K}\left(t, \sigma_{1}\right)-\mathcal{K}\left(t, \sigma_{2}\right)\right\|_{\mathrm{C}^{0}([A, B])}$ in case that $\sigma_{1}, \sigma_{2} \in \mathrm{C}^{0}([A, B])$ :

$$
\left\|\mathcal{K}\left(t, \sigma_{1}\right)-\mathcal{K}\left(t, \sigma_{2}\right)\right\|_{\mathrm{C}^{0}([A, B])} \leq(B-A)\|G\|_{\mathrm{C}^{0}([A, B] \times[A, B])}\left\|\sigma_{1}-\sigma_{2}\right\|_{\mathrm{C}^{0}([A, B])}
$$

The initial interest in introducing operator $\mathcal{K}$ lies in that Problem (1)-(5) can be rewritten as follows: ${ }^{2}$

$$
\left\{\begin{array}{l}
\frac{\mathrm{d} T}{\mathrm{~d} t}=\tilde{f}(t)-c_{1} T^{4}+c_{2} \mathcal{K}\left(t, c_{4} T^{4}\right) \quad \text { on }\left[t_{0}, t_{f}\right] \\
T\left(t_{0}\right)=T_{0} .
\end{array}\right.
$$

\footnotetext{
${ }^{2}$ From now on, due to the functional framework that will be introduced later on, we change the open intervals $\left(t_{0}, t_{f}\right)$ and $(A, B)$ for the closed ones $\left[t_{0}, t_{f}\right]$ and $[A, B]$. Notice that in case (11) holds in the open interval $\left(t_{0}, t_{f}\right)$ for some $T \in$ $\mathrm{C}^{0}\left(\left[t_{0}, t_{f}\right] ; \mathrm{C}^{0}([A, B])\right)$, then (11) will also hold in the closed interval $\left[t_{0}, f_{f}\right]$ provided that $\tilde{f} \in \mathrm{C}^{0}\left(\left[t_{0}, t_{f}\right] ; \mathrm{C}^{0}([A, B])\right)$ and $l_{A}, l_{B} \in \mathrm{C}^{0}\left(\left[t_{0}, t_{f}\right]\right)$. This can be proved by observing that $T \in \mathrm{C}^{1}\left(\left(t_{0}, t_{f}\right) ; \mathrm{C}^{0}([A, B])\right) \cap \mathrm{C}^{0}\left(\left[t_{0}, t_{f}\right] ; \mathrm{C}^{0}([A, B])\right)$ and $\frac{\mathrm{d} T}{\mathrm{~d} t} \in \mathrm{C}^{0}\left(\left[t_{0}, t_{f}\right] ; \mathrm{C}^{0}([A, B])\right)$.
} 
The reader can find in [4], for the $\mathrm{L}^{1}$ setting, theorems of comparison between usual derivatives like $\partial T / \partial t$ an vector derivatives like $\mathrm{d} T / \mathrm{d} t$. We will employ in this paper the fact that

$$
\frac{\mathrm{d} T}{\mathrm{~d} t}(t)(x)=\frac{\partial T}{\partial t}(t, x) \quad \forall(t, x) \in\left[t_{0}, t_{f}\right] \times[A, B]
$$

when $T \in \mathrm{C}^{1}\left(\left[t_{0}, t_{f}\right] ; \mathrm{C}^{0}([A, B])\right)$, by application of the following Lemma.

Lemma 1 Consider a function

$$
\psi:(t, x) \in\left[t_{0}, t_{f}\right] \times[A, B] \longrightarrow \psi(t, x) \in \mathbb{R},
$$

which is continuous with respect to $x$ on $[A, B]$, and define

$$
\hat{\psi}:\left[t_{0}, t_{f}\right] \longrightarrow \mathrm{C}^{0}([A, B])
$$

by means of

$$
\hat{\psi}(t)(x)=\psi(t, x) \quad \forall(t, x) \in\left[t_{0}, t_{f}\right] \times[A, B] .
$$

Suppose that $\hat{\psi}$ is strongly differentiable at some $t_{1} \in\left[t_{0}, t_{f}\right]$. Then $\psi$ is differentiable with respect to $t$ at $t_{1}$, and

$$
\frac{\partial \psi}{\partial t}\left(t_{1}, x\right)=\frac{\mathrm{d} \hat{\psi}}{\mathrm{d} t}\left(t_{1}\right)(x) \quad \forall x \in[A, B] .
$$

PROOF. The result follows from

$$
\begin{gathered}
\lim _{h \rightarrow 0}\left\|\frac{\mathrm{d} \hat{\psi}}{\mathrm{d} t}\left(t_{1}\right)-\frac{\hat{\psi}\left(t_{1}+h\right)-\hat{\psi}\left(t_{1}\right)}{h}\right\|_{\mathrm{C}^{0}([A, B])}=0, \\
\left|\frac{\mathrm{d} \hat{\psi}}{\mathrm{d} t}\left(t_{1}\right)(x)-\frac{\psi\left(t_{1}+h, x\right)-\psi\left(t_{1}, x\right)}{h}\right| \leq\left\|\frac{\mathrm{d} \hat{\psi}}{\mathrm{d} t}\left(t_{1}\right)-\frac{\hat{\psi}\left(t_{1}+h\right)-\hat{\psi}\left(t_{1}\right)}{h}\right\|_{\mathrm{C}^{0}([A, B])},
\end{gathered}
$$

and the uniqueness of limit.

\subsection{The self-adjoint operator $\mathcal{L}$}

Let us define a linear operator $\mathcal{L}: \mathrm{L}^{2}(A, B) \rightarrow \mathrm{L}^{2}(A, B)$ by means of the following equality:

$$
\mathcal{L}(\sigma)(x)=\int_{A}^{B} G(x, s) \sigma(s) \mathrm{d} s, \quad \text { for } x \in[A, B] .
$$

By comparison with Equation (9), it is clear that

$$
\mathcal{K}(t, \sigma)(x)=\mathcal{L}(\sigma)(x)+h(t, x) \quad \text { if } \sigma \in \mathrm{L}^{2}(A, B) .
$$

$\mathcal{L}$ is a Hilbert-Schmidt integral operator with symmetric real kernel $G \in \mathrm{C}^{0}([A, B] \times[A, B])$; consequently, $\mathcal{L}$ is compact and self-adjoint ([9]).

Moreover, the following theorem holds:

Theorem 1 Let $\left\{\mu_{1}, \varphi_{1}\right\} \in \mathbb{R} \times \mathrm{C}^{\infty}([A, B])$ be the first eigenpair of operator $\mathcal{L}$. Then $\mu_{1}>0, \varphi_{1}(x)>0$ $\forall x \in[A, B]$, and $\|\mathcal{L}\|_{\mathrm{L}^{2} \rightarrow \mathrm{L}^{2}}=\mu_{1}$.

Proof. See Appendix B. 


\section{Existence and uniqueness for a truncated version of the problem}

Let us consider, for $M>0$ and $\delta>0$, both arbitrarily fixed, the real function $\eta_{M} \in \mathrm{C}^{0}(\mathbb{R})$ whose graphic is the one depicted in Figure 1. That is to say, for $s \in \mathbb{R}$ :

$$
\eta_{M}(s)= \begin{cases}\frac{s}{M \delta}+\frac{(1+\delta)}{\delta} & \text { if } s \in[-M(1+\delta),-M), \\ 1 & \text { if } s \in[-M, M] \\ -\frac{s}{M \delta}+\frac{(1+\delta)}{\delta} & \text { if } s \in(M, M(1+\delta)] \\ 0 & \text { otherwise. }\end{cases}
$$

The reader can immediately check that

$$
\left|\eta_{M}\left(s_{1}\right)-\eta_{M}\left(s_{2}\right)\right| \leq \frac{1}{M \delta}\left|s_{1}-s_{2}\right| \quad \forall s_{1}, s_{2} \in \mathbb{R} .
$$

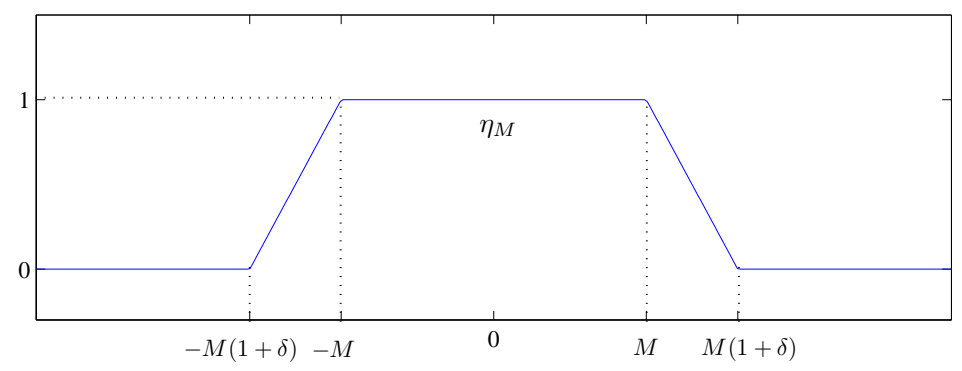

Figure 1. Graphic of the function $\eta_{M}$.

To begin, one can give rapid response to the existence and uniqueness question for the following truncated problem:

$$
\left\{\begin{array}{l}
\frac{\mathrm{d} T}{\mathrm{~d} t}=\tilde{f}(t)-c_{1} \eta_{M}\left(\|T(t)\|_{\mathrm{C}^{0}([A, B])}\right) T^{4}+c_{2} \mathcal{K}\left(t, c_{4} \eta_{M}\left(\|T(t)\|_{\mathrm{C}^{0}([A, B])}\right) T^{4}\right) \quad \text { on }\left[t_{0}, t_{f}\right], \\
T\left(t_{0}\right)=T_{0} .
\end{array}\right.
$$

It is clear that a solution $T \in \mathrm{C}^{1}\left(\left[t_{0}, t_{f}\right] ; \mathrm{C}^{0}([A, B])\right)$ of Problem (13) is also a solution of Problem (11) in case that

$$
\|T(t)\|_{\mathrm{C}^{0}([A, B])} \leq M \quad \text { for all } t \in\left[t_{0}, t_{f}\right] .
$$

This solution, defined for every $t$, would be global. In case there exists $t_{1} \in\left(t_{0}, t_{f}\right]$ such that

$$
\|T(t)\|_{\mathrm{C}^{0}([A, B])} \leq M \quad \text { for all } t \in\left[t_{0}, t_{1}\right]
$$

we would still have a local solution of Problem (11).

Theorem 2 (Existence and uniqueness for Problem (13)) Let us suppose that

1. $T_{0} \in \mathrm{C}^{0}([A, B])$, 
2. $l_{A}, l_{B} \in \mathrm{C}^{0}\left(\left[t_{0}, t_{f}\right]\right)$, and

3. $\tilde{f} \in \mathrm{C}^{0}\left(\left[t_{0}, t_{f}\right] \times[A, B]\right)$.

Then there exists a unique $T \in \mathrm{C}^{1}\left(\left[t_{0}, t_{f}\right] ; \mathrm{C}^{0}([A, B])\right)$ solution of Problem (13).

Corollary 1 (Uniqueness for Problem (11)) Assume that the hypotheses of Theorem 2 hold. Then there exists at most a unique $T \in \mathrm{C}^{1}\left(\left[t_{0}, t_{f}\right] ; \mathrm{C}^{0}([A, B])\right)$ solution of Problem (11).

Proof. The solution of Problem (13) depends on $M$, and in this proof it is convenient to indicate that dependence with a subscript. Moreover, $\|\cdot\|_{C^{0}}$ will stand for $\left.\|\cdot\|_{C^{0}\left(\left[t_{0}, t_{f}\right] \times[A, B]\right.}\right)$.

Let $T \in \mathrm{C}^{1}\left(\left[t_{0}, t_{f}\right] ; \mathrm{C}^{0}([A, B])\right)$ be a solution of Problem (11) and apply Theorem 2 with $M \geq\|T\|_{C^{0}}$ to infer that $T_{M}=T$ for any of those values of $M$. Thus, $T_{M}$ does not depend on $M$ for $M$ large enough when Problem (11) has some solution.

Now, if $T$ and $\hat{T} \in \mathrm{C}^{1}\left(\left[t_{0}, t_{f}\right] ; \mathrm{C}^{0}([A, B])\right)$ are two solutions of Problem (11), we have $T=\hat{T}=T_{M}$ for any $M \geq \max \left\{\|T\|_{C^{0}},\|\hat{T}\|_{C^{0}}\right\}$.

\subsection{Proof of Theorem 2}

The following Theorem 3 is a generalized version, performed in order to include in operator $H$ the dependence on $t$, of the Cauchy-Lipschitz-Picard Theorem as it appears in [1, Ch. VII].

Theorem 3 Let us consider a Banach space $\left(E,\|\cdot\|_{E}\right)$, and let

$$
H:\left[t_{0}, t_{f}\right] \times E \longrightarrow E
$$

be a continuous operator such that

$$
\|H(t, u)-H(t, v)\|_{E} \leq L\|u-v\|_{E} \quad \forall t \in\left[t_{0}, t_{f}\right], \quad \forall u, v \in E,
$$

where $L \in \mathbb{R}$ is independent of $t$.

Then, for all $u_{0} \in E$, there exists a unique $u \in \mathrm{C}^{1}\left(\left[t_{0}, t_{f}\right] ; E\right)$ such that

$$
\left\{\begin{array}{l}
\frac{\mathrm{d} u}{\mathrm{~d} t}=H(t, u) \quad \text { on }\left[t_{0}, t_{f}\right], \\
u\left(t_{0}\right)=u_{0} .
\end{array}\right.
$$

PROOF. We only sketch the proof because it is analogous to that written in [1, Ch. VII]. Furthermore, this sketch is of interest for an eventual numerical resolution by application of the fixed point method.

For given $k>0$, the Bielecki norm on $\mathrm{C}^{0}\left(\left[t_{0}, t_{f}\right] ; E\right)$ defined by

$$
\|u\|_{k}=\sup _{t_{0} \leq t \leq t_{f}}\left\{\mathrm{e}^{-k t}\|u(t)\|_{E}\right\}
$$

is equivalent to the usual one

$$
\|u\|=\sup _{t_{0} \leq t \leq t_{f}}\|u(t)\|_{E},
$$

and consequently $\left(\mathrm{C}^{0}\left(\left[t_{0}, t_{f}\right] ; E\right),\|\cdot\|_{k}\right)$ is a Banach space.

The proof is finished by noting that the mapping

$$
\Phi:\left(\mathrm{C}^{0}\left(\left[t_{0}, t_{f}\right] ; E\right),\|\cdot\|_{k}\right) \longrightarrow\left(\mathrm{C}^{0}\left(\left[t_{0}, t_{f}\right] ; E\right),\|\cdot\|_{k}\right)
$$

defined by

is contractive when $k>L$.

$$
(\Phi u)(t)=u_{0}+\int_{t_{0}}^{t} H(s, u(s)) \mathrm{d} s
$$

Theorem 2 holds in virtue of Theorem 3 and the following Lemma. 
Lemma 2 Assume that the hypotheses of Theorem 2 hold. Then the operator $H:\left[t_{0}, t_{f}\right] \times \mathrm{C}^{0}([A, B]) \rightarrow$ $\mathrm{C}^{0}([A, B])$, defined by

$$
H(t, u)=\tilde{f}(t)-c_{1} \eta_{M}\left(\|u\|_{\mathrm{C}^{0}([A, B])}\right) u^{4}+c_{2} \mathcal{K}\left(t, c_{4} \eta_{M}\left(\|u\|_{\mathrm{C}^{0}([A, B])}\right) u^{4}\right),
$$

is continuous and satisfies (16).

PRoof. Since the continuity of $H$ under the hypotheses of Theorem 2 is easily derived from the continuity of function $\eta_{M}$ and the definition of operator $\mathcal{K}$, we center our attention in proving the Lipschitz property (16). We shall omit the subindex $\mathrm{C}^{0}([A, B])$ in the norm. First note that, due to (10), it suffices to check Lipschitz property for $H^{\star} u=\eta_{M}(\|u\|) u^{4}$.

Obviously, $H^{\star} u_{1}-H^{\star} u_{2}=0$ if $\left\|u_{1}\right\| \geq M(1+\delta)$ and $\left\|u_{2}\right\| \geq M(1+\delta)$.

For general $u_{1}$ and $u_{2}$ we have

$$
\begin{array}{r}
H^{\star} u_{1}-H^{\star} u_{2}=\eta_{M}\left(\left\|u_{1}\right\|\right)\left(u_{1}^{4}-u_{2}^{4}\right)+\left[\eta_{M}\left(\left\|u_{1}\right\|\right)-\eta_{M}\left(\left\|u_{2}\right\|\right)\right] u_{2}^{4}, \\
\text { and } \quad u_{1}^{4}-u_{2}^{4}=\left(u_{1}^{3}+u_{1}^{2} u_{2}+u_{1} u_{2}^{2}+u_{2}^{3}\right)\left(u_{1}-u_{2}\right) .
\end{array}
$$

Consequently,

$$
\left\|H^{\star} u_{1}-H^{\star} u_{2}\right\| \leq\left\{4 \eta_{M}\left(\left\|u_{1}\right\|\right) \max \left(\left\|u_{1}\right\|^{3},\left\|u_{2}\right\|^{3}\right)+\frac{1}{M \delta}\left\|u_{2}\right\|^{4}\right\}\left\|u_{1}-u_{2}\right\| .
$$

One now derives from (17) that, in case that $\left\|u_{1}\right\| \geq M(1+\delta)$ and $\left\|u_{2}\right\|<M(1+\delta)$ (or vice versa, as the roles of $u_{1}$ and $u_{2}$ in (17) can be interchanged),

$$
\left\|H^{\star} u_{1}-H^{\star} u_{2}\right\| \leq \frac{M^{3}(1+\delta)^{4}}{\delta}\left\|u_{1}-u_{2}\right\|,
$$

and that, if $\left\|u_{1}\right\|<M(1+\delta)$ and $\left\|u_{2}\right\|<M(1+\delta)$,

$$
\left\|H^{\star} u_{1}-H^{\star} u_{2}\right\| \leq\left\{4+\frac{(1+\delta)}{\delta}\right\} M^{3}(1+\delta)^{3}\left\|u_{1}-u_{2}\right\|,
$$

which ends the proof, since, according to the previous discussions, the bound obtained in Equation (18) is actually valid for all $u_{1}, u_{2} \in \mathrm{C}^{0}([A, B])$.

Remark 1 (Optimum value of $\delta$ ) When demonstrating Lemma 2, we showed that

$$
L^{\star}=\left\{4+\frac{(1+\delta)}{\delta}\right\} M^{3}(1+\delta)^{3}
$$

is a Lipschitz constant for $H^{\star}$. Elemental calculus shows that, for $M$ given, the minimum value of $L^{\star}=(432 / 25) M^{3}$ is attained when $\delta=1 / 5$. The reader can easily check that

$$
L=\left[c_{1}+(B-A)\|G\|_{\mathrm{C}^{0}([A, B] \times[A, B])} c_{2} c_{4}\right] L^{\star}
$$

is a Lipschitz constant for $H$. In Appendix $A$ it is explained how to compute the value of $\|G\|_{\mathrm{C}^{0}([A, B] \times[A, B])}$.

\section{Existence, uniqueness, bounds}

Assume that the hypotheses of Theorem 2 are satisfied.

Suppose that

$$
T_{0}(x)>0 \quad \forall x \in[A, B]
$$


and take $M>\left\|T_{0}\right\|_{\mathrm{C}^{0}([A, B])}$ in order to have

$$
0<T_{0}(x)<M \quad \forall x \in[A, B] .
$$

Let

$$
T \in \mathrm{C}^{1}\left(\left[t_{0}, t_{f}\right] ; \mathrm{C}^{0}([A, B])\right)
$$

be the unique solution of Problem (13).

Naturally, we must look for conditions on the data ensuring that $T$ satisfies (14), to have a global solution, or (15), to have a local one. Since $T$ stands for temperature in the problem of origin, and thus it must be positive, we will actually look for hypotheses guaranteeing the stronger condition

$$
0 \leq T(t, x) \leq M \quad \forall(t, x) \in\left[t_{0}, t_{f}\right] \times[A, B]
$$

for global solution or

$$
0 \leq T(t, x) \leq M \quad \forall(t, x) \in\left[t_{0}, t_{1}\right] \times[A, B]
$$

for some $t_{1} \in\left(t_{0}, t_{f}\right]$, for a local one.

Define

$$
t_{1}=\inf \left\{t \in\left[t_{0}, t_{f}\right]: t=t_{f} \quad \text { or } \quad\|T(t)\|_{C^{0}([A, B])}=M \quad \text { or } \quad \min _{A \leq x \leq B} T(t, x)=0\right\} .
$$

By employing arguments of continuity one proves that $t_{1}$ exists in $\left(t_{0}, t_{f}\right]$ and that $0 \leq T(t, x) \leq M$ $\forall(t, x) \in\left[t_{0}, t_{1}\right] \times[A, B]$. Hence (22) is guaranteed, and we have the following theorem.

Theorem 4 (Local existence and uniqueness for Problem (11)) Assume that the hypotheses of Theorem 2, and hypothesis (19) hold. Then there exists a unique $T \in \mathrm{C}^{1}\left(\left[t_{0}, t_{1}\right] ; \mathrm{C}^{0}([A, B])\right)$ solution of Problem (11) on $\left[t_{0}, t_{1}\right]$, for certain $t_{1} \in\left(t_{0}, t_{f}\right]$.

On the other hand, condition (21) holds if $t_{1}=t_{f}$.

Set now the additional hypotheses

$$
\begin{aligned}
l_{A}(t) & \geq 0, l_{B}(t) \geq 0 & & \forall t \in\left[t_{0}, t_{1}\right], \\
\tilde{f}(t, x) & \geq 0 & & \forall(t, x) \in\left[t_{0}, t_{1}\right] \times[A, B] .
\end{aligned}
$$

Notice that, since $c_{4} T^{4} \geq 0$ is guaranteed, hypothesis (24) implies

$$
\mathcal{K}\left(t, c_{4} T^{4}\right) \geq 0 .
$$

Then one can prove the following theorem.

Theorem 5 (Lower bound for the local solution of Problem (11)) Assume that the hypotheses of Theorem 2, and hypotheses (19), (24), and (25) hold. Let T be the unique local solution of Problem (11) derived from Theorem 4 . Then

$$
T(t, x) \geq \frac{T_{0}(x)}{\sqrt[3]{3 c_{1}\left(t-t_{0}\right) T_{0}^{3}(x)+1}} \quad \forall(t, x) \in\left[t_{0}, t_{1}\right] \times[A, B] .
$$

Proof. Take into account that

$$
\begin{cases}\frac{\partial T}{\partial t}(t, x)=\tilde{f}(t, x)-c_{1} T^{4}(t, x)+c_{2} \mathcal{K}\left(t, c_{4} T^{4}\right)(x) \geq-c_{1} T^{4}(t, x) & \forall(t, x) \in\left[t_{0}, t_{1}\right] \times[A, B], \\ T\left(t_{0}, x\right)=T_{0}(x) & \forall x \in[A, B],\end{cases}
$$

and apply the following Lemma 3. 
Remark 2 In particular, $T>0$ under the hypotheses of Theorem 5.

Lemma 3 Let $y:\left[t_{0}, t_{1}\right] \rightarrow \mathbb{R}$ be an absolutely continuous function such that $y\left(t_{0}\right)=y_{0}>0$. Let $N \subset\left[t_{0}, t_{1}\right]$ be the set of points at which $y$ is not differentiable (therefore $N$ has zero measure). Assume that, for certain constant $C>0$,

$$
y^{\prime}(t) \geq-C y^{4}(t) \quad \forall t \in\left[t_{0}, t_{1}\right] \backslash N .
$$

Then

$$
y(t) \geq \frac{y_{0}}{\sqrt[3]{3 C\left(t-t_{0}\right) y_{0}^{3}+1}} \quad \forall t \in\left[t_{0}, t_{1}\right]
$$

ProOF. Set

$$
t^{\star}=\sup \left\{\bar{t} \in\left(t_{0}, t_{1}\right]: y(t)>0 \quad \forall t \in\left[t_{0}, \bar{t}\right]\right\} .
$$

Integrating

$$
\frac{\mathrm{d}}{\mathrm{d} t}\left(y^{-3}(t)\right)=-3 y^{-4}(t) y^{\prime}(t) \leq 3 C \quad \forall t \in\left[t_{0}, t^{\star}\right) \backslash N
$$

we infer

or, equivalently,

$$
\frac{1}{y^{3}(t)}-\frac{1}{y_{0}^{3}} \leq 3 C\left(t-t_{0}\right) \quad \forall t \in\left[t_{0}, t^{\star}\right)
$$

$$
y^{3}(t) \geq \frac{y_{0}^{3}}{3 C\left(t-t_{0}\right) y_{0}^{3}+1} \quad \forall t \in\left[t_{0}, t^{\star}\right) .
$$

By continuity, inequality (27) also holds for $t=t^{\star}$, and consequently $y\left(t^{\star}\right)>0$ and $t^{\star}=t_{1}$.

Lemma 4 Assume that the hypotheses of Theorem 5 hold. Then time $t_{1}$, defined by Equation (23), is also defined by the following equality:

$$
t_{1}=\inf \left\{t \in\left[t_{0}, t_{f}\right]: t=t_{f} \text { or }\|T(t)\|_{C^{0}([A, B])}=M\right\} .
$$

Proof. From inequality (26) we deduce that $\min _{x \in[A, B]} T\left(x, t_{1}\right)>0$, which ends the proof.

Previous Lemma 4 shows that $t_{1}=t_{f}$ is ensured if

$$
\left\|T\left(t_{1}\right)\right\|_{\mathrm{C}^{0}([A, B])}<M,
$$

which in turn holds in case that (recall that $T$ depends on $M$ )

$$
\left\|T\left(t_{1}\right)\right\|_{\mathrm{C}^{0}([A, B])} \leq K \text {, being } K \text { a constant independent of } M \text {. }
$$

Indeed, if (28) holds we could take $M>\max \left\{K,\left\|T_{0}\right\|_{\mathrm{C}^{0}([A, B])}\right\}$ from the beginning in order to reach this point of the reasoning with the certainty that $\left\|T\left(t_{1}\right)\right\|_{\mathrm{C}^{0}([A, B])}<M$.

Now the study bifurcates depending upon the sign of

$$
\gamma=c_{2} c_{4} \mu_{1}-c_{1} .
$$

When $\gamma>0$, we will show that (28) cannot be guaranteed; when $\gamma \leq 0$, which includes the interesting case from the physical point of view, we will prove (28), which as we know implies global solution.

Given two real and measurable functions $\psi_{1}$ and $\psi_{2}$ defined almost everywhere on $[A, B]$, we will use the notation

$$
\left(\psi_{1}, \psi_{2}\right)=\int_{A}^{B} \psi_{1}(x) \psi_{2}(x) \mathrm{d} x
$$

whenever the integral exists in $\mathbb{R}$. It is clear that $(\cdot, \cdot)$ stands for the inner product in $\mathrm{L}^{2}(A, B)$ in case that $\psi_{1}$ and $\psi_{2}$ belong to $\mathrm{L}^{2}(A, B)$.

Recall that $\left\{\mu_{1}, \varphi_{1}\right\} \in \mathbb{R} \times \mathrm{C}^{\infty}([A, B])$ is the first eigenpair of operator $\mathcal{L}$ defined in Subsection 2.2. 


\subsection{Case $\gamma>0$}

Assume that the hypotheses of Theorem 5 hold, and recall who is $T$ from (20) and adjacent lines.

Lemma 5 For any $\varphi \in \mathrm{L}^{1}(A, B)$,

$$
\frac{\mathrm{d}}{\mathrm{d} t}(T(t), \varphi)=\left(\frac{\partial T}{\partial t}(t), \varphi\right)
$$

PROOF. The proof is easy by employing dominated convergence or, in a more elementary way, by using the chain rule to derive the composition $L \circ T$, being $L: \mathrm{C}^{0}([A, B]) \rightarrow \mathbb{R}$ the linear and continuous mapping defined by $L(T)=(T, \varphi)$ for $T \in \mathrm{C}^{0}([A, B])$.

Previous Lemma 5 justifies the following equality:

$$
\frac{\mathrm{d}}{\mathrm{d} t}\left(T(t), \varphi_{1}\right)=\left(\tilde{f}(t), \varphi_{1}\right)-c_{1}\left(T^{4}(t), \varphi_{1}\right)+c_{2} c_{4}\left(\mathcal{L}\left(T^{4}(t)\right), \varphi_{1}\right)+c_{2}\left(h(t), \varphi_{1}\right) \quad \forall t \in\left[t_{0}, t_{1}\right]
$$

Since $\mathcal{L}$ is self-adjoint, we have

$$
\left(\mathcal{L}\left(T^{4}(t)\right), \varphi_{1}\right)=\left(T^{4}(t), \mathcal{L}\left(\varphi_{1}\right)\right)=\mu_{1}\left(T^{4}(t), \varphi_{1}\right)
$$

and consequently Equation (30) can be rewritten as follows:

$$
\frac{\mathrm{d}}{\mathrm{d} t}\left(T(t), \varphi_{1}\right)=\left(\tilde{f}(t)+c_{2} h(t), \varphi_{1}\right)+\gamma\left(T^{4}(t), \varphi_{1}\right) \quad \forall t \in\left[t_{0}, t_{1}\right]
$$

with $\gamma$ defined by Equation (29).

Lemma 6 Let $\mu^{\star}$ be a positive measure on $(A, B)$. Then

$$
\left(\int_{A}^{B} u \mathrm{~d} \mu^{\star}\right)^{4} \leq\left(\mu^{\star}\{(A, B)\}\right)^{3} \int_{A}^{B} u^{4} \mathrm{~d} \mu^{\star} \quad \forall u \in \mathrm{L}^{4}\left((A, B), \mu^{\star}\right) .
$$

PROOF. By Hölder's inequality,

$$
\left|\int_{A}^{B} u \mathrm{~d} \mu^{\star}\right| \leq\left(\int_{A}^{B} u^{4} \mathrm{~d} \mu^{\star}\right)^{1 / 4}\left(\int_{A}^{B} 1 \mathrm{~d} \mu^{\star}\right)^{3 / 4}
$$

which ends the proof.

Application of Lemma 6 with $\mu^{\star}=\varphi_{1} \mathrm{~d} x$ shows that

$$
\left(T^{4}(t), \varphi_{1}\right) \geq\left(\int_{A}^{B} \varphi_{1}(x) \mathrm{d} x\right)^{-3}\left(T(t), \varphi_{1}\right)^{4},
$$

and then Equation (31) implies

$$
\frac{\mathrm{d}}{\mathrm{d} t}\left(T(t), \varphi_{1}\right) \geq \gamma\left(\int_{A}^{B} \varphi_{1}(x) \mathrm{d} x\right)^{-3}\left(T(t), \varphi_{1}\right)^{4} \quad \forall t \in\left[t_{0}, t_{1}\right]
$$

if $\gamma>0$. 
Lemma 7 Let $y:\left[t_{0}, t_{1}\right] \rightarrow \mathbb{R}$ be an absolutely continuous function such that $y\left(t_{0}\right)=y_{0}>0$. Let $N \subset\left[t_{0}, t_{1}\right]$ be the set of points at which $y$ is not differentiable (therefore $N$ has zero measure). Assume that, for certain constant $C>0$,

$$
y^{\prime}(t) \geq C y^{4}(t) \quad \forall t \in\left[t_{0}, t_{1}\right] \backslash N .
$$

Then

$$
y(t) \geq \frac{y_{0}}{\sqrt[3]{1-3 C\left(t-t_{0}\right) y_{0}^{3}}} \quad \forall t \in\left[t_{0}, t_{1}\right]
$$

and, consequently,

$$
t_{1}<t^{*}=t_{0}+\frac{1}{3 C y_{0}^{3}} .
$$

Proof. It is clear that $y(t)>0$ for all $t \in\left[t_{0}, t_{1}\right]$, since $y_{0}>0$ and $y^{\prime} \geq 0$. Then

$$
\frac{\mathrm{d}}{\mathrm{d} t}\left(-y^{-3}(t)\right)=3 y^{-4}(t) y^{\prime}(t) \geq 3 C \quad \forall t \in\left[t_{0}, t_{1}\right] .
$$

Integrating between $t_{0}$ and $t$ one obtains

$$
y^{3}(t) \geq v(t)=\frac{y_{0}^{3}}{1-3 C\left(t-t_{0}\right) y_{0}^{3}} \quad \forall t \in\left[t_{0}, t_{1}\right]
$$

which ends the proof, as $\lim _{t \uparrow t^{*}} v(t)=+\infty$.

Theorem 6 (Barrier and second lower bound for the local solution of Problem (11)) Assume that the hypotheses of Theorem 5 hold, and let $T$ be the unique local solution of Problem (11) derived from Theorem 4. Assume also that $\gamma>0$, with $\gamma$ defined by Equation (29). Then

$$
\left(T(t), \varphi_{1}\right) \geq b(t)=\frac{\left(T_{0}, \varphi_{1}\right)}{\sqrt[3]{1-3 \gamma\left(1, \varphi_{1}\right)^{-3}\left(t-t_{0}\right)\left(T_{0}, \varphi_{1}\right)^{3}}} \quad \forall t \in\left[t_{0}, t_{1}\right]
$$

and, consequently,

$$
t_{1}<t^{*}=t_{0}+\frac{1}{3 \gamma}\left[\frac{\left(1, \varphi_{1}\right)}{\left(T_{0}, \varphi_{1}\right)}\right]^{3}
$$

Proof. Apply Lemma 7 taking into account inequality (32).

Remark 3 As a consequence of Theorem 6 we have for sure that the solution cannot be global when $t^{*} \leq t_{f}$.

\subsection{Case $\gamma \leq 0$}

Assume that the hypotheses of Theorem 5 hold, and recall who is $T$ from (20) and adjacent lines.

If $\gamma \leq 0$, Equation (31) imply

$$
\frac{\mathrm{d}}{\mathrm{d} t}\left(T(t), \varphi_{1}\right) \leq\left(\tilde{f}(t)+c_{2} h(t), \varphi_{1}\right) \quad \forall t \in\left[t_{0}, t_{1}\right] .
$$

Notice the contrast with the situation for $\gamma>0$, analyzed in Theorem 6 , because integration of inequality (34) provides us with the following upper bound for $\left(T(t), \varphi_{1}\right)$ :

$$
\left(T(t), \varphi_{1}\right) \leq\left(T_{0}, \varphi_{1}\right)+\int_{t_{0}}^{t}\left(\tilde{f}(s)+c_{2} h(s), \varphi_{1}\right) \mathrm{d} s \quad \forall t \in\left[t_{0}, t_{1}\right] .
$$


Going beyond, in this case one can actually prove condition (28).

In virtue of Theorem 5 , we have, for any value of $\gamma \in \mathbb{R}$,

$$
\begin{aligned}
\frac{\partial T}{\partial t}(t, x) & =\tilde{f}(t, x)+c_{2} h(t, x)+c_{2} c_{4} \mathcal{L}\left(T^{4}(t)\right)(x)-c_{1} T^{4}(t, x) \\
& \leq \tilde{f}(t, x)+c_{2} h(t, x)+c_{2} c_{4} \mathcal{L}\left(T^{4}(t)\right)(x)-\frac{c_{1} T_{0}^{4}(x)}{\left(3 c_{1}\left(t-t_{0}\right) T_{0}^{3}(x)+1\right)^{4 / 3}}
\end{aligned}
$$

for all $(t, x) \in\left[t_{0}, t_{1}\right] \times[A, B]$.

Consequently, one can obtain an a priori upper bound of $\frac{\partial T}{\partial t}$ in case one gets an upper bound of

$$
\mathcal{L}\left(T^{4}(t)\right)(x)=\int_{A}^{B} G(x, s) T^{4}(t, s) \mathrm{d} s,
$$

which in turn is done if one gets an upper bound of $T^{4}(t)$ in any $\mathrm{L}^{p}(A, B)$ norm, $p \geq 1$.

As demonstrated in Subsection 4.1, one cannot expect to find that bound when $\gamma>0$. On the contrary, in the case $\gamma \leq 0$ we shall obtain an upper bound of $\left\|T^{4}(t)\right\|_{L^{5 / 4}(A, B)}$.

Note that

$$
\left\|T^{4}(t)\right\|_{\mathrm{L}^{5 / 4}(A, B)}=\|T(t)\|_{\mathrm{L}^{5}(A, B)}^{4} .
$$

Since $T$ is positive one has, by dominated convergence,

$$
\frac{\mathrm{d}}{\mathrm{d} t}\left(\|T(t)\|_{\mathrm{L}^{5}(A, B)}^{5}\right)=5\left(\frac{\partial T}{\partial t}(t), T^{4}(t)\right),
$$

and then

$$
\begin{aligned}
\|T(t)\|_{\mathrm{L}^{5}(A, B)}^{4} & \frac{\mathrm{d}}{\mathrm{d} t}\left(\|T(t)\|_{\mathrm{L}^{5}(A, B)}\right)=\frac{1}{5} \frac{\mathrm{d}}{\mathrm{d} t}\left(\|T(t)\|_{\mathrm{L}^{5}(A, B)}^{5}\right) \\
& =\left(\tilde{f}(t)+c_{2} h(t), T^{4}(t)\right)+c_{2} c_{4}\left(\mathcal{L}\left(T^{4}(t)\right), T^{4}(t)\right)-c_{1}\left\|T^{4}(t)\right\|_{\mathrm{L}^{2}(A, B)}^{2} \\
& \leq\left(\tilde{f}(t)+c_{2} h(t), T^{4}(t)\right)+\gamma\left\|T^{4}(t)\right\|_{\mathrm{L}^{2}(A, B)}^{2} \quad \forall t \in\left[t_{0}, t_{1}\right],
\end{aligned}
$$

where it has been taken into account that

$$
\begin{aligned}
\left(\mathcal{L}\left(T^{4}(t)\right), T^{4}(t)\right) & \leq\left\|\mathcal{L}\left(T^{4}(t)\right)\right\|_{\mathrm{L}^{2}(A, B)}\left\|T^{4}(t)\right\|_{\mathrm{L}^{2}(A, B)} \\
& \leq\|\mathcal{L}\|_{\mathrm{L}^{2} \rightarrow \mathrm{L}^{2}}\left\|T^{4}(t)\right\|_{\mathrm{L}^{2}(A, B)}^{2} \\
& =\mu_{1}\left\|T^{4}(t)\right\|_{\mathrm{L}^{2}(A, B)}^{2} .
\end{aligned}
$$

When $\gamma \leq 0$, inequality (37) implies

$$
\begin{aligned}
\|T(t)\|_{\mathrm{L}^{5}(A, B)}^{4} \frac{\mathrm{d}}{\mathrm{d} t}\left(\|T(t)\|_{\mathrm{L}^{5}(A, B)}\right) \\
\leq\left(\tilde{f}(t)+c_{2} h(t), T^{4}(t)\right) \\
\leq\left\|\tilde{f}(t)+c_{2} h(t)\right\|_{\mathrm{L}^{5}(A, B)}\left\|T^{4}(t)\right\|_{\mathrm{L}^{\frac{5}{4}}(A, B)} \quad \forall t \in\left[t_{0}, t_{1}\right], \\
\quad=\left\|\tilde{f}(t)+c_{2} h(t)\right\|_{\mathrm{L}^{5}(A, B)}\|T(t)\|_{\mathrm{L}^{5}(A, B)}^{4} \quad \forall t
\end{aligned}
$$

from where, since $T>0$,

$$
\frac{\mathrm{d}}{\mathrm{d} t}\left(\|T(t)\|_{\mathrm{L}^{5}(A, B)}\right) \leq\left\|\tilde{f}(t)+c_{2} h(t)\right\|_{\mathrm{L}^{5}(A, B)} \quad \forall t \in\left[t_{0}, t_{1}\right] .
$$


Therefore, for all $t \in\left[t_{0}, t_{1}\right]$,

$$
\|T(t)\|_{\mathrm{L}^{5}(A, B)} \leq\left\|T_{0}\right\|_{\mathrm{L}^{5}(A, B)}+\int_{t_{0}}^{t}\left\|\tilde{f}(s)+c_{2} h(s)\right\|_{\mathrm{L}^{5}(A, B)} \mathrm{d} s .
$$

Now we can obtain an upper bound of $\left\|\mathcal{L}\left(T^{4}(t)\right)\right\|_{\mathrm{C}^{0}([A, B])}$, which in turn allows one to give successively upper bounds of $\frac{\partial T}{\partial t}$ and $T$, and finally state the main result.

\subsubsection{Bound of $\left\|\mathcal{L}\left(T^{4}(t)\right)\right\|_{\mathrm{C}^{0}([A, B])}$.}

From Equations (12), (36) and (38) it is easy to check that

$$
\begin{aligned}
\left\|\mathcal{L}\left(T^{4}(t)\right)\right\|_{\mathrm{C}^{0}([A, B])} & \leq K^{*}\left\|T^{4}(t)\right\|_{\mathrm{L}^{5 / 4}(A, B)} \\
& \leq K^{*}\left(\left\|T_{0}\right\|_{\mathrm{L}^{5}(A, B)}+\int_{t_{0}}^{t}\left\|\tilde{f}(s)+c_{2} h(s)\right\|_{\mathrm{L}^{5}(A, B)} \mathrm{d} s\right)^{4} \quad \forall t \in\left[t_{0}, t_{1}\right]
\end{aligned}
$$

being

$$
K^{*}=\max _{x \in[A, B]}\left(\int_{A}^{B}[G(x, s)]^{5} \mathrm{~d} s\right)^{1 / 5}
$$

\subsubsection{Bounds of $\partial T / \partial t, T$, and main result.}

Let us define

$$
U(t)=\left(\left\|T_{0}\right\|_{\mathrm{L}^{5}(A, B)}+\int_{t_{0}}^{t}\left\|\tilde{f}(s)+c_{2} h(s)\right\|_{\mathrm{L}^{5}(A, B)} \mathrm{d} s\right)^{4} \quad \forall t \in\left[t_{0}, t_{1}\right]
$$

and

$$
w(t, x)=\frac{T_{0}(x)}{\sqrt[3]{3 c_{1}\left(t-t_{0}\right) T_{0}^{3}(x)+1}} \quad \forall(t, x) \in\left[t_{0}, t_{1}\right] \times[A, B] .
$$

Now Equations (35) and (39) imply

$$
\frac{\partial T}{\partial t}(t, x) \leq \tilde{f}(t, x)+c_{2} h(t, x)+c_{2} c_{4} K^{*} U(t)-c_{1} w^{4}(t, x)
$$

for all $(t, x) \in\left[t_{0}, t_{1}\right] \times[A, B]$, from where we derive, by integration,

$$
\begin{aligned}
T(t, x) & \leq T_{0}(x)+\int_{t_{0}}^{t}\left\{\tilde{f}(s, x)+c_{2} h(s, x)+c_{2} c_{4} K^{*} U(s)-c_{1} w^{4}(s, x)\right\} \mathrm{d} s \\
& =w(t, x)+\int_{t_{0}}^{t}\left\{\tilde{f}(s, x)+c_{2} h(s, x)+c_{2} c_{4} K^{*} U(s)\right\} \mathrm{d} s
\end{aligned}
$$

for all $(t, x) \in\left[t_{0}, t_{1}\right] \times[A, B]$.

To compact writing, let us define, for $(t, x) \in\left[t_{0}, t_{f}\right] \times[A, B]$,

$$
\begin{gathered}
\phi(t, x)=\int_{t_{0}}^{t}\left\{\tilde{f}(s, x)+c_{2} h(s, x)+c_{2} c_{4} K^{*} U(s)\right\} \mathrm{d} s, \\
\Psi(t, x)=w(t, x)+\phi(t, x) .
\end{gathered}
$$

Thus we have proved (28) with

$$
K=\|\Psi\|_{\mathrm{C}^{0}\left(\left[t_{0}, t_{f}\right] \times[A, B]\right)}
$$

and the following theorem holds. 
Theorem 7 (Existence, uniqueness and bounds for Problem (11)) Assume that the hypotheses of Theorem 5 hold, consider some $M>K$, with $K$ defined by Equation (44)), and let $T$ be the unique solution of Problem (13) derived from Theorem 2. Assume also that $\gamma \leq 0$, with $\gamma$ defined by Equation (29). Then $T$ is also the unique global solution of Problem (11) and, moreover,

$$
w(t, x) \leq T(t, x) \leq w(t, x)+\phi(t, x) \quad \forall(t, x) \in\left[t_{0}, t_{f}\right] \times[A, B],
$$

where $w$ and $\phi$ are defined by Equations (41) and (43)), respectively.

Proof. Note that $\Psi\left(t_{0}, x\right)=T_{0}(x)$, which implies that $\left\|T\left(t_{1}\right)\right\|_{\mathrm{C}^{0}([A, B])}<M$ (and hence $t_{1}=t_{f}$ ) is ensured when $T$ is the solution of Problem (13) for any $M>K$ (see Equation (28) and lines below).

The inequality $w \leq T$ comes from Theorem 5 , and $T \leq w+\phi$ is exactly the inequality (42).

Remark 4 In order to give a simpler (but coarser) upper bound for T, take into account that

$$
\|u\|_{\mathrm{L}^{5}(A, B)} \leq(B-A)^{1 / 5}\|u\|_{\mathrm{C}^{0}([A, B])}
$$

if $u$ is continuous on $[A, B]$ to deduce from (45) that, under the hypotheses of Theorem 7 ,

$$
\begin{aligned}
\|T\|_{\mathrm{C}^{0}} \leq \| & T_{0} \|_{\mathrm{C}^{0}([A, B])}+\left(t_{f}-t_{0}\right)\left\{\|\tilde{f}\|_{\mathrm{C}^{0}}+c_{2}\|h\|_{\mathrm{C}^{0}}+\right. \\
& \left.\quad+c_{2} c_{4} K^{*}(B-A)^{\frac{4}{5}}\left(\left\|T_{0}\right\|_{\mathrm{C}^{0}([A, B])}+\left(t_{f}-t_{0}\right)\left(\|\tilde{f}\|_{\mathrm{C}^{0}}+c_{2}\|h\|_{\mathrm{C}^{0}}\right)\right)^{4}\right\},
\end{aligned}
$$

where $\|\cdot\|_{\mathrm{C}^{0}}$ stands for $\|\cdot\|_{\mathrm{C}^{0}\left(\left[t_{0}, t_{f}\right] \times[A, B]\right)}$ and $K^{*}$ is the constant defined in Equation (40).

\section{Refining the upper bound when $\gamma<0$}

Assume that the hypotheses of Theorem 7 hold, and let us suppose that $\gamma<0$.

We have seen in Subsection 4.2 how to have an upper bound for $\|T(t)\|_{L^{5}(A, B)}$ like that obtained in Equation (38) allows one to obtain an upper bound for $T$ like the one stated in Theorem 7 . We are going to refine the upper bound for $T$ by refining the bound (38).

Equation (37) implies

$$
\|T(t)\|_{\mathrm{L}^{5}(A, B)}^{4} \frac{\mathrm{d}}{\mathrm{d} t}\left(\|T(t)\|_{\mathrm{L}^{5}(A, B)}\right) \leq\left(\tilde{f}(t)+c_{2} h(t), T^{4}(t)\right)-|\gamma|\left\|T^{4}(t)\right\|_{\mathrm{L}^{2}(A, B)}^{2} \quad \forall t \in\left[t_{0}, t_{f}\right] .
$$

Since in virtue of Hölder's inequality

$$
\left(\tilde{f}(t)+c_{2} h(t), T^{4}(t)\right) \leq\left\|\tilde{f}(t)+c_{2} h(t)\right\|_{\mathrm{L}^{5}(A, B)}\|T(t)\|_{\mathrm{L}^{5}(A, B)}^{4}
$$

and

$$
\|T(t)\|_{\mathrm{L}^{5}(A, B)}^{5} \leq(B-A)^{3 / 8}\left(\left\|T^{4}(t)\right\|_{\mathrm{L}^{2}(A, B)}^{2}\right)^{5 / 8},
$$

we deduce from inequality (46) that

$$
\frac{\mathrm{d}}{\mathrm{d} t}\left(\|T(t)\|_{\mathrm{L}^{5}(A, B)}\right) \leq\left\|\tilde{f}(t)+c_{2} h(t)\right\|_{\mathrm{L}^{5}(A, B)}-|\gamma|(B-A)^{-3 / 5}\|T(t)\|_{\mathrm{L}^{5}(A, B)}^{4}
$$

for all $t \in\left[t_{0}, t_{f}\right]$. 
Lemma 8 Let $y:\left[t_{0}, t_{f}\right] \rightarrow \mathbb{R}$ be an absolutely continuous function. Let $N \subset\left[t_{0}, t_{f}\right]$ be the set of points at which $y$ is not differentiable (therefore $N$ has zero measure). Assume that, for certain real constants $C>0$, $p>0$, and certain nonnegative continuous functions $\delta:\left[t_{0}, t_{f}\right] \rightarrow[0, \infty)$ and $\beta:\left[t_{0}, t_{f}\right] \rightarrow[0, \infty)$, the following two inequalities are satisfied:

$$
y(t) \geq \beta(t) \quad \forall t \in\left[t_{0}, t_{f}\right]
$$

and

$$
y^{\prime}(t)+C y^{p}(t) \leq \delta(t) \quad \forall t \in\left[t_{0}, t_{f}\right] \backslash N .
$$

Then, for all $t \in\left[t_{0}, t_{f}\right]$,

$$
y(t) \leq \min \left\{y\left(t_{0}\right)+\int_{t_{0}}^{t}\left[\delta(s)-C \beta^{p}(s)\right] \mathrm{d} s, \max \left\{y\left(t_{0}\right),\left(\frac{\delta_{\max }(t)}{C}\right)^{1 / p}\right\}\right\},
$$

where $\delta_{\max }(t)=\max _{t_{0} \leq s \leq t} \delta(s)$.

Proof. The inequality

$$
y(t) \leq y\left(t_{0}\right)+\int_{t_{0}}^{t}\left[\delta(s)-C \beta^{p}(s)\right] \mathrm{d} s
$$

is easily inferred from

$$
y^{\prime}(t) \leq \delta(t)-C y^{p}(t) \leq \delta(t)-C \beta^{p}(t)
$$

Now we will show that

$$
y(t) \leq \max \left\{y\left(t_{0}\right),\left(\frac{\delta_{\max }(t)}{C}\right)^{1 / p}\right\} \quad \forall t \in\left[t_{0}, t_{f}\right] .
$$

It suffices to prove that

$$
y(t) \leq \max \left\{y\left(t_{0}\right),\left(\frac{\delta(t)}{C}\right)^{\frac{1}{p}}\right\} \quad \forall t \in\left[t_{0}, t_{f}\right]
$$

when $\delta$ is nondecreasing.

To this end, we note that, if $\delta$ is nondecreasing and there exists $\bar{t} \in\left[t_{0}, t_{f}\right)$ such that $y(\bar{t}) \leq(\delta(\bar{t}) / C)^{1 / p}$, then $y(t) \leq(\delta(t) / C)^{1 / p}$ for all $t \in\left[\bar{t}, t_{f}\right]$. This can be seen through a proof by contradiction: suppose that $y(\tau)>(\delta(\tau) / C)^{1 / p}$ for some $\tau \in\left(\bar{t}, t_{f}\right]$ and define

$$
t_{1}=\sup \left\{t \in[\bar{t}, \tau]: y(t) \leq\left(\frac{\delta(t)}{C}\right)^{1 / p}\right\} .
$$

Continuity of involved functions makes true the relationships $t_{1}<\tau$ and $y\left(t_{1}\right)=\left(\delta\left(t_{1}\right) / C\right)^{1 / p}$. Moreover, it is clear from the definition of $t_{1}$ that $y(t)>(\delta(t) / C)^{1 / p}$ for all $t \in\left(t_{1}, \tau\right]$, which implies $y^{\prime}(t)<0$ on $\left(t_{1}, \tau\right] \backslash N$ owing to hypothesis (48). Previous steps justify the chain

$$
\left(\frac{\delta(\tau)}{C}\right)^{1 / p}<y(\tau)<y\left(t_{1}\right)=\left(\frac{\delta\left(t_{1}\right)}{C}\right)^{1 / p}
$$

which cannot happen, since $\delta$ is nondecreasing.

The proof is completed by considering the following two cases:

- If $y\left(t_{0}\right) \leq\left(\delta\left(t_{0}\right) / C\right)^{1 / p}$, then $y(t) \leq(\delta(t) / C)^{1 / p}$ for all $t \in\left[t_{0}, t_{f}\right]$ and hence (49) is satisfied. 
- If $y\left(t_{0}\right)>\left(\delta\left(t_{0}\right) / C\right)^{1 / p}$, let us define

$$
t_{1}=\sup \left\{t \in\left[t_{0}, t_{f}\right]: y(s)>\left(\frac{\delta(s)}{C}\right)^{1 / p} \forall s \in\left[t_{0}, t\right]\right\} .
$$

Note that hypothesis (48) implies that $y(t)$ is decreasing on $\left[t_{0}, t_{1}\right]$. Thus, (49) is obviously satisfied if $t_{1}=t_{f}$. If $t_{1}<t_{f}$, then we have $y(t)<y\left(t_{0}\right)$ for all $t \in\left(t_{0}, t_{1}\right]$ and $y\left(t_{1}\right)=\left(\delta\left(t_{1}\right) / C\right)^{1 / p}$, which as we proved before obliges to $y(t) \leq(\delta(t) / C)^{1 / p}$ for all $t \in\left[t_{1}, t_{f}\right]$, and consequently (49) holds again.

According to Equation (45), $T(x, t) \geq w(x, t)$. Thus,

$$
\|T(t)\|_{\mathrm{L}^{5}(A, B)} \geq\|w(t)\|_{\mathrm{L}^{5}(A, B)} \quad \forall t \in\left[t_{0}, t_{f}\right]
$$

and we can state the following lemma, which improves the upper bound obtained in Equation (38).

Lemma 9 (Fine upper bound for $\|T(t)\|_{\mathrm{L}^{5}(A, B)}$ ) Assume that the hypotheses of Theorem 7 hold, and let $T$ be the unique global solution of Problem (11) derived from that theorem. Assume also that $\gamma<0$, with $\gamma$ defined by Equation (29). Then

$$
\|T(t)\|_{\mathrm{L}^{5}(A, B)} \leq \Theta^{*}(t) \quad \forall t \in\left[t_{0}, t_{f}\right],
$$

being

$$
\Theta^{*}(t)=\min \left\{\left\|T_{0}\right\|_{\mathrm{L}^{5}(A, B)}+\int_{t_{0}}^{t}\left[\delta(s)-C \beta^{4}(s)\right] \mathrm{d} s, \max \left\{\left\|T_{0}\right\|_{\mathrm{L}^{5}(A, B)},\left(\frac{\delta_{\max }(t)}{C}\right)^{1 / 4}\right\}\right\}
$$

where in turn

$$
\begin{gathered}
\delta(t)=\left\|\tilde{f}(t)+c_{2} h(t)\right\|_{\mathrm{L}^{5}(A, B)}, \\
\delta_{\max }(t)=\max _{t_{0} \leq s \leq t} \delta(s) \\
\beta(t)=\|w(t)\|_{\mathrm{L}^{5}(A, B)}, \quad \text { with } w \text { defined by Equation }(41),
\end{gathered}
$$

and

$$
C=|\gamma|(B-A)^{-3 / 5} .
$$

PROOF. The result is a direct application of Lemma 8 taking into account Equations (47) and (50))

Now the same arguments employed in Subsection 4.2 show that the following theorem holds.

Theorem 8 (Fine upper bound for the solution of Problem (11)) Assume that the hypotheses of Theorem 7 hold, and let $T$ be the unique global solution of Problem (11) derived from that theorem. Assume also that $\gamma<0$, with $\gamma$ defined by Equation (29). Then

$$
w(t, x) \leq T(t, x) \leq w(t, x)+\phi^{*}(t, x) \quad \forall(t, x) \in\left[t_{0}, t_{f}\right] \times[A, B],
$$

where $w$ is defined by Equation (41) and

$$
\phi^{*}(t, x)=\int_{t_{0}}^{t}\left\{\tilde{f}(s, x)+c_{2} h(s, x)+c_{2} c_{4} K^{*}\left(\Theta^{*}(s)\right)^{4}\right\} \mathrm{d} s,
$$

with $K^{*}$ and $\Theta^{*}$ defined by Equations (40) and (51), respectively. 


\section{Appendix A The Green function $G$ and the function $h$}

Following the reference [2], one can compute the Green function $G$ and the function $h$ associated with the boundary value problem (6).

Define

$$
k^{*}=2\left\{\left(c_{3} c_{5}^{2}+1\right) \sinh \left[(B-A) \sqrt{c_{3}}\right]+2 \sqrt{c_{3}} c_{5} \cosh \left[(B-A) \sqrt{c_{3}}\right]\right\} .
$$

The Green function is given by the following expressions (Equations (53) and (54)):

If $A \leq s \leq x \leq B$,

$$
\begin{array}{r}
G(x, s)=\frac{1}{2 \sqrt{c_{3}} k^{*}}\left\{( 1 - \sqrt { c _ { 3 } } c _ { 5 } ) \left[\left(1-\sqrt{c_{3}} c_{5}\right) \mathrm{e}^{-\sqrt{c_{3}}(B-A-x+s)}\right.\right. \\
\left.-\left(1+\sqrt{c_{3}} c_{5}\right) \mathrm{e}^{\sqrt{c_{3}}(B+A-x-s)}\right] \\
+\left(1+\sqrt{c_{3}} c_{5}\right)\left[\left(1+\sqrt{c_{3}} c_{5}\right) \mathrm{e}^{\sqrt{c_{3}}(B-A-x+s)}\right. \\
\left.\left.-\left(1-\sqrt{c_{3}} c_{5}\right) \mathrm{e}^{-\sqrt{c_{3}}(B+A-x-s)}\right]\right\} .
\end{array}
$$

If $A \leq x \leq s \leq B$,

$$
\begin{aligned}
G(x, s)=\frac{1}{2 \sqrt{c_{3}} k^{*}}\left\{( 1 + \sqrt { c _ { 3 } } c _ { 5 } ) \left[\left(1+\sqrt{c_{3}} c_{5}\right) \mathrm{e}^{\sqrt{c_{3}}(B-A+x-s)}\right.\right. \\
\left.-\left(1-\sqrt{c_{3}} c_{5}\right) \mathrm{e}^{-\sqrt{c_{3}}(B+A-x-s)}\right] \\
+\left(1-\sqrt{c_{3}} c_{5}\right)\left[\left(1-\sqrt{c_{3}} c_{5}\right) \mathrm{e}^{-\sqrt{c_{3}}(B-A+x-s)}\right. \\
\left.\left.-\left(1+\sqrt{c_{3}} c_{5}\right) \mathrm{e}^{\sqrt{c_{3}}(B+A-x-s)}\right]\right\} .
\end{aligned}
$$

Notice that $G$ is symmetric, in the sense that $G(x, s)=G(s, x)$ for all $(x, s) \in[A, B] \times[A, B]$. This property evinces that $G$ is continuous on the diagonal segment $D=\{(x, x): x \in[A, B]\}$, and hence on $[A, B] \times[A, B]$.

The reader can check that, for $\bar{x}$ fixed in $(A, B]$, the function $\omega(s)=G(\bar{x}, s)$ satisfies $\omega^{\prime}(s) \geq 0$ for all $s \in[A, \bar{x}]$. Hence, due to the symmetry of $G$, one has, on one hand, that $G$ is nonnegative because $G(\bar{x}, A) \geq 0$ and, on the other hand, that the maximum of $G$ on the diagonal segment $D$ is equal to the maximum of $G$ on $[A, B] \times[A, B]$ (that is to say, to $\|G\|_{\mathrm{C}^{0}([A, B] \times[A, B])}$ ). Moreover, it is easy to check that $G(A, A)=G(B, B)$.

By differentiating the function $g(x)=G(x, x), x \in[A, B]$, we obtain

$$
g^{\prime}(x)=\frac{\left(1-c_{3} c_{5}^{2}\right)}{k^{*}}\left\{\mathrm{e}^{\sqrt{c_{3}}(B+A-2 x)}-\mathrm{e}^{-\sqrt{c_{3}}(B+A-2 x)}\right\},
$$

and, consequently, the following assertions hold:

- If $c_{3} c_{5}^{2}=1$, the maximum of $G$ on $[A, B] \times[A, B]$ is equal to $G(x, x)$ for any $x \in[A, B]$.

- If $c_{3} c_{5}^{2}<1$, the maximum of $G$ on $[A, B] \times[A, B]$ is equal to $G(c, c)$, being $c=(A+B) / 2$ the center of $[A, B]$.

- If $c_{3} c_{5}^{2}>1$, the maximum of $G$ on $[A, B] \times[A, B]$ is equal to $G(A, A)$ or, equivalently, to $G(B, B)$.

In Figures 2 and 3 we can observe the graphic of the Green function corresponding to particular choices of $A, B, c_{3}$, and $c_{5}$. 


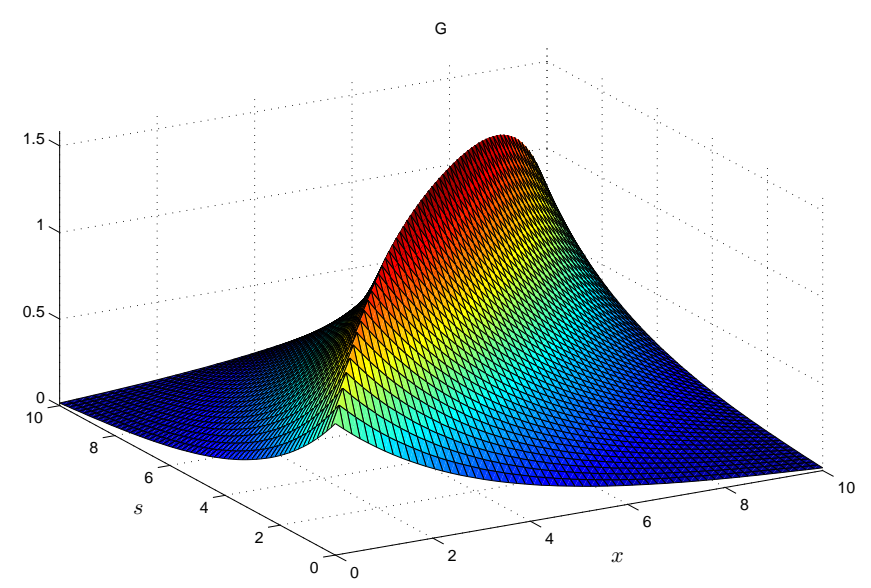

Figure 2. Graphic of the Green function $G$ when $A=0, B=10, c_{3}=0.1$, and $c_{5}=1$. Note that $c_{3} c_{5}^{2}<1$.

We finish this section with the expression of the function $h$ :

$$
\begin{aligned}
h(x)=\frac{2}{k^{*}} & \left\{\left[\sinh \left(\sqrt{c_{3}}(x-A)\right)+\sqrt{c_{3}} c_{5} \cosh \left(\sqrt{c_{3}}(x-A)\right)\right] l_{B}\right. \\
+ & {\left.\left[\sinh \left(\sqrt{c_{3}}(B-x)\right)+\sqrt{c_{3}} c_{5} \cosh \left(\sqrt{c_{3}}(B-x)\right)\right] l_{A}\right\} . }
\end{aligned}
$$

Thus, for $(t, x) \in\left[t_{0}, t_{f}\right] \times[A, B]$, we have

$$
\begin{aligned}
h(t, x)=\frac{2}{k^{*}} & \left\{\left[\sinh \left(\sqrt{c_{3}}(x-A)\right)+\sqrt{c_{3}} c_{5} \cosh \left(\sqrt{c_{3}}(x-A)\right)\right] l_{B}(t)\right. \\
+ & {\left.\left[\sinh \left(\sqrt{c_{3}}(B-x)\right)+\sqrt{c_{3}} c_{5} \cosh \left(\sqrt{c_{3}}(B-x)\right)\right] l_{A}(t)\right\} . }
\end{aligned}
$$

\section{Appendix B The first eigenpair $\left\{\mu_{1}, \varphi_{1}\right\}$}

Operator $\mathcal{L}: \mathrm{L}^{2}(A, B) \rightarrow \mathrm{L}^{2}(A, B)$ defined by Equation (12) can also be defined, equivalently, as follows: $u=\mathcal{L}(\sigma)$ (we will use equivalently the notation $\mathcal{L} \sigma$ ) is the unique solution of the boundary value problem

$$
\left\{\begin{array}{l}
-u^{\prime \prime}+c_{3} u=\sigma \quad \text { on }(A, B), \\
u(A)-c_{5} u^{\prime}(A)=0, \\
u(B)+c_{5} u^{\prime}(B)=0 .
\end{array}\right.
$$

Since $\mathcal{L}$ is self-adjoint, all its eigenvalues are real. Also, for a given eigenvalue, real eigenfunctions exist in $\mathrm{C}^{\infty}([A, B])$ and the following definition is valid.

Definition $1\{\mu, \varphi\} \in \mathbb{R} \times \mathrm{C}^{\infty}([A, B])$ is said to be an eigenpair of $\mathcal{L}$ in case that $\varphi \not \equiv 0$ and $\mathcal{L} \varphi=\mu \varphi$. The scalar $\mu$ is called an eigenvalue of $\mathcal{L}$ and the function $\varphi$ is called an eigenfunction of $\mathcal{L}$ associated with the eigenvalue $\mu$.

We notice that 0 is not an eigenvalue of $\mathcal{L}$, because $\mathcal{L} \varphi=0$ only when $\varphi \equiv 0$. Thus, $\{\mu, \varphi\} \in$ $\mathbb{R} \times \mathrm{C}^{\infty}([A, B])$ is an eigenpair of $\mathcal{L}$ if, and only if, $\mu \neq 0, \varphi \neq \equiv$ and

$$
\left\{\begin{array}{l}
-\varphi^{\prime \prime}+c_{3} \varphi=\lambda \varphi \quad \text { on }(A, B), \\
\varphi(A)-c_{5} \varphi^{\prime}(A)=0, \\
\varphi(B)+c_{5} \varphi^{\prime}(B)=0
\end{array}\right.
$$




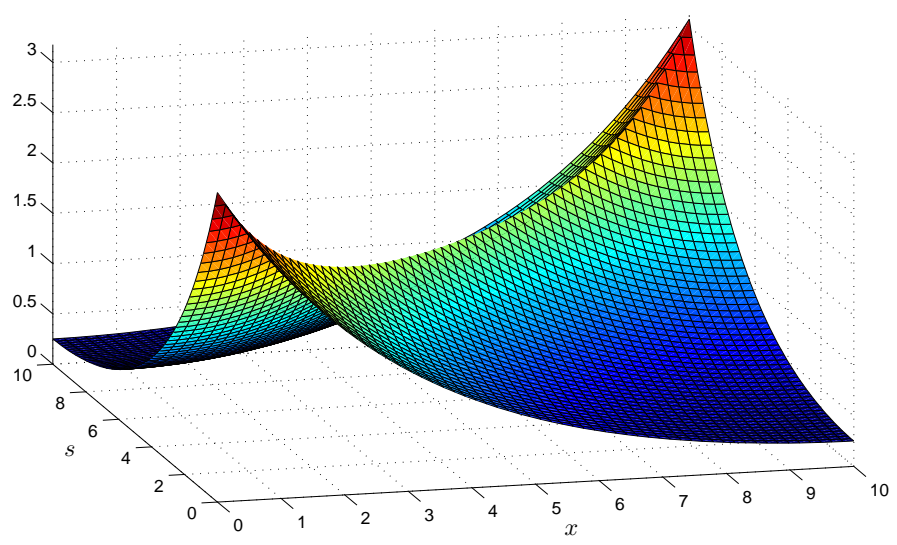

Figure 3. Graphic of the Green function $G$ when $A=0, B=10, c_{3}=0.1$, and $c_{5}=100$. Note that $c_{3} c_{5}^{2}>1$.

with $\lambda=1 / \mu$.

Since Problem (55) has only the trivial solution $\varphi \equiv 0$ when $c_{3}-\lambda \geq 0$ (recall Equation (6) and adjacent lines), the eigenvalues $\mu=1 / \lambda$ must satisfy $c_{3}-\lambda<0$. That is to say,

$$
\text { if } \mu \text { is an eigenvalue of } \mathcal{L} \text {, then } 0<\mu<\frac{1}{c_{3}} \text {. }
$$

On the other hand, if $\left\{\mu_{i}, \varphi_{i}\right\}$ and $\left\{\mu_{j}, \varphi_{j}\right\}$ are eigenpairs of $\mathcal{L}$, then

$$
\mu_{i}\left(\varphi_{i}, \varphi_{j}\right)=\left(\mathcal{L} \varphi_{i}, \varphi_{j}\right)=\left(\varphi_{i}, \mathcal{L} \varphi_{j}\right)=\mu_{j}\left(\varphi_{i}, \varphi_{j}\right),
$$

which proves that $\left(\varphi_{i}, \varphi_{j}\right)=0$ when $\mu_{i} \neq \mu_{j}$; that is to say, self-adjointness implies that two eigenfunctions associated with two distinct eigenvalues are orthogonal.

\section{Appendix B.1 Changing to $[0, \pi]$}

Let us take $\lambda>c_{3}$. Looking for the simplest calculus, we perform a change of variable in order to transform Problem (55) into the following one:

$$
\left\{\begin{array}{l}
-\psi^{\prime \prime}+\tilde{c}_{3} \psi=\tilde{\lambda} \psi \quad \text { on }(0, \pi), \\
\psi(0)-\tilde{c}_{5} \psi^{\prime}(0)=0 \\
\psi(\pi)+\tilde{c}_{5} \psi^{\prime}(\pi)=0
\end{array}\right.
$$

where

$$
\tilde{c}_{3}=c_{3} \frac{(B-A)^{2}}{\pi^{2}}, \quad \tilde{\lambda}=\lambda \frac{(B-A)^{2}}{\pi^{2}}, \quad \tilde{c}_{5}=c_{5} \frac{\pi}{(B-A)} .
$$

Given a solution $\psi$ of (57), the following function $\varphi$ is a solution of (55):

$$
\varphi(x)=\psi\left[\frac{\pi}{(B-A)}(x-A)\right] \quad \text { for } x \in[A, B],
$$

and all the solutions of (55) can be obtained in this way. 
The solutions of the ODE $-\psi^{\prime \prime}+\tilde{c}_{3} \psi=\tilde{\lambda} \psi$ are of the form

$$
\psi(x)=K_{1} \sin (p x)+K_{2} \cos (p x)
$$

with $K_{1}$ and $K_{2}$ real constants and $p=\sqrt{\tilde{\lambda}-\tilde{c}_{3}}>0$. One can easily check that the boundary conditions are satisfied if, and only if, constants $K_{1}$ and $K_{2}$ obey the following equations:

$$
\begin{gathered}
K_{2}=\tilde{c}_{5} p K_{1}, \\
K_{2} \frac{\left(1-\tilde{c}_{5}^{2} p^{2}\right)}{\tilde{c}_{5} p} \sin (p \pi)+2 K_{2} \cos (p \pi)=0 .
\end{gathered}
$$

If $K_{2}=0$, then $K_{1}=0$ and $\psi \equiv 0$. If $K_{2} \neq 0$, the second equation in (58) is equivalent to

$$
\frac{\left(1-\tilde{c}_{5}^{2} p^{2}\right)}{\tilde{c}_{5} p} \sin (p \pi)+2 \cos (p \pi)=0
$$

Thus, Problem (57) with $\tilde{\lambda}=\tilde{c}_{3}+p^{2}$, being $p>0$, has nonzero solutions if, and only if, Equation (59) holds.

Now note that:

- $p \in(0, \infty)$ such that $\cos (p \pi)=0$ solves Equation (59) if, and only if, $\tilde{c}_{5} p=1$. In this circumstance

$$
\psi_{K}(x)=K\left(\sin \left(\frac{1}{\tilde{c}_{5}} x\right)+\cos \left(\frac{1}{\tilde{c}_{5}} x\right)\right), \quad x \in[0, \pi], K \in \mathbb{R}
$$

are all the solutions of Problem (57) for $\tilde{\lambda}=\tilde{c}_{3}+1 / \tilde{c}_{5}^{2}$. We can encounter this situation only when $\tilde{c}_{5}=2 /\left(1+2 k^{\star}\right)$ for some $k^{\star} \in \mathbb{N} \cup\{0\}$.

- $p \in(0, \infty)$ such that $\cos (p \pi) \neq 0$ solves Equation (59) if, and only if,

$$
\tan (p \pi)=\frac{2 \tilde{c}_{5} p}{\tilde{c}_{5}^{2} p^{2}-1}, \quad p \in(0, \infty) \backslash\left\{\frac{1}{\tilde{c}_{5}}\right\},
$$

which has an unbounded denumerable set of solutions. We encounter this situation for any value $\tilde{c}_{5} \in(0, \infty)$.

\section{Appendix B.2 Eigenpairs of $\mathcal{L}$}

According to the previous analysis, the eigenvalues of operator $\mathcal{L}$, in decreasing order, are exhaustively described by:

$$
\mu_{i}=\frac{1}{\tilde{\lambda}_{i}} \frac{(B-A)^{2}}{\pi^{2}}, \quad i \in \mathbb{N}
$$

where $\tilde{\lambda}_{i}=\tilde{c}_{3}+p_{i}^{2}$, and the sequence $\left\{p_{i}\right\}_{i \in \mathbb{N}} \subset(0, \infty)$ is as follows:

- If $\tilde{c}_{5} \in(0, \infty) \backslash\{2 /(1+2 k): k \in \mathbb{N} \cup\{0\}\},\left\{p_{i}\right\}_{i \in \mathbb{N}}$ is the set of solutions of Equation (60) in increasing order.

- If $\tilde{c}_{5}=2 /\left(1+2 k^{\star}\right)$ for some $k^{\star} \in \mathbb{N} \cup\{0\}$, then $\left\{p_{i}\right\}_{i \in \mathbb{N} \backslash\left\{k^{\star}+1\right\}}$ is the set of solutions of Equation (60) in increasing order, and $p_{k^{\star}+1}=1 / 2+k^{\star}$.

Since $\left\{p_{i}\right\}_{i \in \mathbb{N}}$ is an increasing sequence that tends to infinity, the sequence of eigenvalues $\left\{\mu_{i}\right\}_{i \in \mathbb{N}}$ is decreasing and converges to zero. Moreover, property (56) can be obtained from Equation (61). 
The eigenfunctions associated with the eigenvalue $\mu_{i}$ are given by

$$
\varphi_{i, K}(x)=\psi_{i, K}\left[\frac{\pi}{(B-A)}(x-A)\right], \quad x \in[A, B], K \in \mathbb{R} \backslash\{0\},
$$

where

$$
\psi_{i, K}(x)=K\left[\sin \left(p_{i} x\right)+\tilde{c}_{5} p_{i} \cos \left(p_{i} x\right)\right], \quad x \in[0, \pi] .
$$

The first or greatest eigenvalue $\mu_{1}$ can be computed by computing $p_{1}$ as follows:

- If $\tilde{c}_{5}>2$, then $p_{1}$ is the unique solution of Equation (60) in $\left(1 / \tilde{c}_{5}, 1 / 2\right)$.

- If $\tilde{c}_{5}=2$, then $p_{1}=1 / 2$. Consequently,

$$
\mu_{1}=\frac{4(B-A)^{2}}{4 c_{3}(B-A)^{2}+\pi^{2}} .
$$

- If $\tilde{c}_{5} \in(0,2)$, then $p_{1}$ is the unique solution of Equation $(60)$ in $\left(1 / 2, \min \left(1 / \tilde{c}_{5}, 1\right)\right)$.

When $\tilde{c}_{5} \in(0, \infty) \backslash\{2\}$, it is easy to implement a Newton-Raphson algorithm ensuring convergence to the solution $p_{1}$.

The first eigenfunction $\varphi_{1}$ used in the paper, and mentioned in Theorem 1 , is any of the eigenfunctions $\varphi_{1, K}$ with $K \in(0, \infty)$, as the results do not depend on the constant $K$ but $\varphi_{1}$ must be positive. Clearly, the positivity of $\varphi_{1}$ on $[A, B]$ is equivalent to the positivity of $\psi_{1,1}$ on $[0, \pi]$ :

$$
\psi_{1,1}(x)=\sin \left(p_{1} x\right)+\tilde{c}_{5} p_{1} \cos \left(p_{1} x\right), \quad x \in[0, \pi] .
$$

The next proposition ends the proof of Theorem 1.

Proposition 1 The following assertions hold:

1. $\varphi_{1}(x)>0$ for all $x \in[A, B]$.

2. $\|\mathcal{L}\|_{L^{2} \rightarrow L^{2}}=\mu_{1}$.

PROOF.

1. We will show that $\psi_{1,1}(x)>0$ for all $x \in[0, \pi]$. This is immediate if $\tilde{c}_{5} \geq 2$, since then $p_{1} \leq 1 / 2$.

Let us suppose that $\tilde{c}_{5} \in(0,2)$, which implies $p_{1} \in\left(1 / 2, \min \left(1 / \tilde{c}_{5}, 1\right)\right)$. Then it is clear that $\psi_{1,1}(x)>0$ for all $x \in\left[0, \pi /\left(2 p_{1}\right)\right]$. To analyze the sign on $\left(\pi /\left(2 p_{1}\right), \pi\right]$, notice that $\psi_{1,1}^{\prime}$ is negative on that interval and that $\psi_{1,1}(\pi)>0$. Indeed, taking into account that $\cos \left(p_{1} \pi\right)<0$ and $\tan \left(p_{1} \pi\right)=\left(2 \tilde{c}_{5} p_{1}\right) /\left(\tilde{c}_{5}^{2} p_{1}^{2}-1\right)$, one can see that $\psi_{1,1}(\pi)>0$ is satisfied if, and only if, $\left(\tilde{c}_{5}^{2} p_{1}^{2}+1\right) /\left(\tilde{c}_{5}^{2} p_{1}^{2}-1\right)<0$, which holds because $0<\tilde{c}_{5} p_{1}<1$.

2. Recall that

$$
\|\mathcal{L}\|_{\mathrm{L}^{2} \rightarrow \mathrm{L}^{2}}=\sup _{\substack{\sigma \in \mathrm{L}^{2}(A, B) \\\|\sigma\|_{\mathrm{L}^{2}(A, B)} \leq 1}}\|\mathcal{L} \sigma\|_{\mathrm{L}^{2}(A, B)}
$$

One can easily check that $\left\|\varphi_{i, K}\right\|_{\mathrm{L}^{2}(A, B)}^{2}=(B-A) K^{2}\left\|\psi_{i, 1}\right\|_{\mathrm{L}^{2}(0, \pi)}^{2} / \pi$. For given $i \in \mathbb{N}$, let us define the normalized eigenfunction $\varphi_{i}^{N}=\varphi_{i, K^{\star}}$, being $K^{\star}$ the unique in $(0, \infty)$ such that $\left\|\varphi_{i, K^{\star}}\right\|_{\mathrm{L}^{2}(A, B)}=1$. Since $\left\{\varphi_{i}^{N}\right\}_{i \in \mathbb{N}}$ is a Hilbert basis of $\mathrm{L}^{2}(A, B)$ and the sequence of eigenvalues is decreasing, we have, for any $\sigma \in \mathrm{L}^{2}(A, B)$,

$$
\|\mathcal{L} \sigma\|_{\mathrm{L}^{2}(A, B)}^{2}=\sum_{i=1}^{\infty} \mu_{i}^{2}\left|\left(\sigma, \varphi_{i}^{N}\right)\right|^{2} \leq \mu_{1}^{2} \sum_{i=1}^{\infty}\left|\left(\sigma, \varphi_{i}^{N}\right)\right|^{2}=\mu_{1}^{2}\|\sigma\|_{\mathrm{L}^{2}(A, B)}^{2},
$$


which in turn implies $\|\mathcal{L}\|_{\mathrm{L}^{2} \rightarrow \mathrm{L}^{2}} \leq \mu_{1}$.

On the other hand,

$$
\left\|\mathcal{L} \varphi_{1}^{N}\right\|_{L^{2}(A, B)}^{2}=\sum_{i=1}^{\infty} \mu_{i}^{2}\left|\left(\varphi_{1}^{N}, \varphi_{i}^{N}\right)\right|^{2}=\mu_{1}^{2},
$$

from where $\|\mathcal{L}\|_{\mathrm{L}^{2} \rightarrow \mathrm{L}^{2}} \geq \mu_{1}$.

\section{Appendix C Why $\gamma<0$ in the physically relevant case}

First, we establish the relationship between the notations of this paper and those of reference [3], which contains the physically relevant system. See Equations (2.9), (2.14), (3.27), and (3.28) of reference [3] to conclude that $c_{1}=\left(4 a n^{2} \sigma\right) /\left(\rho c_{v}\right), c_{2}=(2 \pi a) /\left(\rho c_{v}\right), c_{3}=(a \alpha) /\left(\mu_{G}^{2}\right), c_{4}=\left(2 a \alpha n^{2} \sigma\right) /\left(\mu_{G}^{2} \pi\right)$, and $c_{5}=\mu_{G} / \alpha$ if we choose $l_{A}(t)=2 I_{+A}(t)$ and $l_{B}(t)=2 I_{-B}(t)$.

Here $\alpha=a+\sigma_{s}$ and $\mu_{G}=1 / \sqrt{3}$.

Take into account that $c_{2} c_{4}=c_{1} c_{3}$ to write

$$
\gamma=c_{2} c_{4} \mu_{1}-c_{1}=c_{1}\left(c_{3} \mu_{1}-1\right) .
$$

Since Equation (61) implies

$$
c_{3} \mu_{1}-1=\frac{-\pi^{2} p_{1}^{2}}{\pi^{2} p_{1}^{2}+3 a \alpha(B-A)^{2}},
$$

one derives from Equation (62) that $\gamma<0$.

By the way, one obtains at the same cost the bound

$$
|\gamma|<c_{1}=\frac{4 a n^{2} \sigma}{\rho c_{v}}
$$

evincing that, in the range of applications, the order of magnitude of $\gamma$ is small due to the presence in the expression of $c_{1}$ of the Stefan-Boltzmann constant $\sigma=5.6696 \times 10^{-8}$ (SI units $\mathrm{W} /\left(\mathrm{m}^{2} \mathrm{~K}^{4}\right)$ ).

On the other hand, from the analysis developed in Appendix B, one can see that

$$
\lim _{(B-A) \uparrow \infty} \gamma=0,
$$

which provides us with the asymptotic behavior of $\gamma$ for large space intervals.

\section{Appendix D Conclusions}

This scholarly article is closely associated to, and in fact it has been motivated by, reference [3], where system (1)-(5) was solved numerically without having a proof of existence or uniqueness of solution. In [3] the values of the constants $c_{i}, i \in\{1,2,3,4,5\}$, were such that the critical parameter $\gamma$ is negative, which in virtue of Theorem 7 ensures existence and uniqueness of global solution $T \in \mathrm{C}^{1}\left(\left[t_{0}, t_{f}\right] ; \mathrm{C}^{0}([A, B])\right)$ under the following hypotheses:

1. $T_{0} \in \mathrm{C}^{0}([A, B])$ and positive,

2. $l_{A}, l_{B} \in \mathrm{C}^{0}\left(\left[t_{0}, t_{f}\right]\right)$, both nonnegative,

3. $\tilde{f} \in \mathrm{C}^{0}\left(\left[t_{0}, t_{f}\right] \times[A, B]\right)$ and nonnegative. 
Continuity of data is quite reasonable in many contexts, although it is easy to think of lacking continuity models; an example is Test 3 of reference [3].

Leaving apart the discussion about continuity, and centering our attention on the sign of the data functions, we notice that the first two hypotheses are natural from the physics, since $T_{0}$ is the initial temperature and $l_{A}$ and $l_{B}$ are, up to a positive multiplicative constant, values of the total intensity of radiation. The third one, regarding $\tilde{f}$, is somewhat more restrictive, since it allows for internal heat sources (or no internal heat supply at all), but it does not for internal heat sinks that could be present, due for instance to some inflow of cold gas or to endothermic chemical reactions taking place inside the domain.

Moreover, the solution $T$ is bounded from below and from above according to Equation (52).

When $\gamma=0$ the problem has still a unique global solution under the same hypotheses, and it is bounded from below and from above according to Equation (45).

In case that $\gamma>0$, and under the same hypotheses, the problem has a unique local solution bounded from below according to Equation (26).

In all three cases $(\gamma<0, \gamma=0$ and $\gamma>0)$ the lower bound is the same, and consequently the positivity of the solution is guaranteed.

The reason why we cannot assure that the solution is global when $\gamma>0$ is as follows: we have seen in Equation (33) that the scalar product of $T$ with the eigenfunction $\varphi_{1}$ is bounded from below by a function which blows up at certain finite time $t^{*}>t_{0}$, which in turn prevents a continuous $T$ from being defined up to and beyond that barrier. Moreover, we have the additional information that $t^{*}$ tends to $t_{0}$ as $\gamma$ goes to infinity.

We should also mention that the adjectives global and local are always referred to the time domain $\left[t_{0}, t_{f}\right]$. With respect to the spatial domain $[A, B]$, all the solutions which we have written about are global.

Although not explicitly said till now, it is evident that one can obtain, from the bounds for $T$, bounds for the radiative heat flux $F$ by making use of the fact that $F=\mathcal{K}\left(t, c_{4} T^{4}\right)$. Regarding its regularity, we notice that, under the hypotheses of existence of this paper, $F$ is continuous in time and of class $\mathrm{C}^{2}$ in space: $F \in \mathrm{C}^{0}\left(\left[t_{0}, t_{f}\right] ; \mathrm{C}^{2}([A, B])\right)$.

With the aim that the critical parameter $\gamma$ and the bounds of the solution can be computed in a practical case with the minimum effort, we have included in the appendices the relevant information about $G, h, \mu_{1}$, and $\varphi_{1}$.

\section{References}

[1] BrÉzIS, H. (2005) Analyse fonctionnelle: théorie et applications, Dunod, Paris (first published by Masson in 1983).

[2] Cabada, A. And Lois, S., (1999). Existence results for nonlinear problems with separated boundary conditions, Nonlinear Anal., 35, 4, 449-456. DOI: 10.1016/S0362-546X(97)00618-4

[3] Falcone, M. And López Pouso, Ó., (2003). Analysis and comparison of two approximation schemes for a radiative transfer system, Math. Models Methods Applísi., 13, 2, 159-186.

[4] López Pouso, Ó., (2003). Comparison between usual and vector time derivatives, Glasg. Math. J., 45, 1, 167172. DOI: $10.1017 /$ S001708950200112X

[5] Modest, M. F., (2003). Radiative heat transfer, Elsevier Science, San Diego, California, USA. (Second edition; first published by McGraw-Hill in 1993).

[6] Pinnau, R., (2007). Analysis of optimal boundary control for radiative heat transfer modeled by the $S P_{1}$-system, Commun. Math. Sci., 5, 4, 951-969.

[7] Porzio, M. M. And López Pouso, Ó., (2004). Application of accretive operators theory to evolutive combined conduction, convection, and radiation, Rev. Mat. Iberoam., 20, 1, 257-275. 
[8] Siegel, R. And Howell, J. R., (2002). Thermal radiation heat transfer, Taylor \& Francis, New York, USA. (fourth edition; first published in 1971).

[9] YosidA, K., (1995). Functional Analysis, Springer, Berlin, Germany. (reprint of the sixth edition for the collection "Springer Classics in Mathematics"; first published in 1965; sixth edition dated 1980)

\section{Óscar López Pouso}

Departamento de Matemática Aplicada, Facultad de Matemáticas,

Universidad de Santiago de Compostela,

C/ Lope Gómez de Marzoa s/n,

Campus Universitario Sur,

15782 Santiago de Compostela (A Coruña),

SPAIN

oscar. lopez@usc.es

\section{Rafael Muñoz Sola}

Departamento de Matemática Aplicada, Facultad de Matemáticas, Universidad de Santiago de Compostela, C/ Lope Gómez de Marzoa s/n, Campus Universitario Sur, 15782 Santiago de Compostela (A Coruña), SPAIN

rafael.munoz@usc.es 